Elsevier Editorial System(tm) for Journal of Molecular Biology

Manuscript Draft

Manuscript Number:

Title: Architecture of the VE-cadherin hexamer

Article Type: Full Length Article

Section/Category: Protein and nucleic acid structure, function, and interactions

Keywords: VE-cadherin; homophilic association; homology model; hexamer; electron microscopy

Corresponding Author: Dr Elizabeth Ann Hewat, DPhil

Corresponding Author's Institution: Laboratoire de Microscopie Electronique Structurale

First Author: Elizabeth Ann Hewat, DPhil

Order of Authors: Elizabeth Ann Hewat, DPhil; Claire Durmort, PhD; Lilian Jacquamet, PhD; Evelyne Concord; Danielle Gulino-Debrac, PhD

Manuscript Region of Origin:

Abstract: Vascular endothelial-cadherin (VE-cadherin) is the major constituent of the adherens junctions of endothelial cells and plays a key role in angiogenesis and vascular permeability. The ectodomains EC1-4 of VE-cadherin are known to form hexamers in solution. To examine the mechanism of homotypic association of VE-cadherin, we have made a 3D reconstruction of the hexamer using electron microscopy and produced a homology model based on the known structure of C-cadherin EC1-5. The hexamer consists of a trimer of dimers with each N-terminal EC1 module making an anti-parallel dimeric contact, and the EC4 modules forming extensive trimeric interactions. Each EC1-4 molecule makes a helical curve allowing some torsional flexibility to the edifice. While there is no direct evidence for the existence of hexamers of cadherin at adherens junctions, the model we have produced provides indirect evidence since it can be used to explain a number of disparate results for adherens junctions. It is in accord with the X-ray and electron microscopy 
results which demonstrate that the EC1 dimer is central to homotypic cadherin interaction. It provides an explanation for the force measurements of the interaction between opposing cadherin layers, which have previously been interpreted as resulting from three different interdigitating interactions. It is in accord with observations of native junctions by cryo-electron microscopy. The fact that this hexameric model of VEcadherin can be used to explain more of the existing data on adherens junctions than any other model alone argues in favour of the existance of the hexamer at the adherens junction. In the context of the cell-cell junction these cis-trimers close to the membrane, and trans-dimers from opposing membranes, would increase the avidity of the bond. 


\section{Architecture of the VE-cadherin hexamer}

Elizabeth A Hewat ${ }^{1}$, Claire Durmort ${ }^{2}$, Lilian Jacquamet ${ }^{3}$, Evelyne Concord ${ }^{2}$ and Danielle Gulino-Debrac ${ }^{2}$.

${ }^{\mathbf{1}}$ Laboratoire de Microscopie Electronique Structurale, ${ }^{\mathbf{2}}$ Laboratoire d'Ingénierie des Macromolécules , ${ }^{\mathbf{3}}$ Laboratoire de Cristallographie et Cristallogenèse des Protéines, Institut de Biologie Structurale Jean-Pierre Ebel, UMR 5075, CEA-CNRS-UJF, 41 rue Jules Horowitz, 38027 Grenoble Cedex 1, France.

Corresponding author:

Elizabeth Hewat: $\quad$ Tel: 33 (0) 438784568

Fax: $33(0) 438785494$

email: hewat@ibs.fr 


\section{Summary}

Vascular endothelial-cadherin (VE-cadherin) is the major constituent of the adherens junctions of endothelial cells and plays a key role in angiogenesis and vascular permeability. The ectodomains EC1 4 of VE-cadherin are known to form hexamers in solution. To examine the mechanism of homotypic association of VE-cadherin, we have made a 3D reconstruction of the hexamer using electron microscopy and produced a homology model based on the known structure of C-cadherin EC1 -5. The hexamer consists of a trimer of dimers with each N-terminal EC1 module making an anti-parallel dimeric contact, and the EC4 modules forming extensive trimeric interactions. Each EC1-4 molecule makes a helical curve allowing some torsional flexibility to the edifice. While there is no direct evidence for the existence of hexamers of cadherin at adherens junctions, the model we have produced provides indirect evidence since it can be used to explain a number of disparate results for adherens junctions. It is in accord with the X-ray and electron microscopy results which demonstrate that the EC1 dimer is central to homotypic cadherin interaction. It provides an explanation for the force measurements of the interaction between opposing cadherin layers, which have previously been interpreted as resulting from three different interdigitating interactions. It is in accord with observations of native junctions by cryo-electron microscopy. The fact that this hexameric model of VE-cadherin can be used to explain more of the existing data on adherens junctions than any other model alone argues in favour of the existance of the hexamer at the adherens junction. In the context of the cell-cell junction these cis-trimers close to the membrane, and trans-dimers from opposing membranes, would increase the avidity of the bond.

Keywords: VE-cadherin; homophilic association; homology model; hexamer; electron microscopy; 


\section{Introduction}

Cadherins constitute a superfamily of $\mathrm{Ca}^{++}$-dependent adhesive molecules that bind cells together via homotypic interactions ${ }^{1}$. By promoting clustering of cells with identical phenotypes, cadherins play a crucial role in the elaboration of various tissues during embryogenesis and in the maintenance of tissue architecture. Junctions between endothelial cells, in particular adherens junctions, rely on the interaction of vascular endothelium (VE) cadherin molecules that are specifically expressed at these junctions. VE-cadherin is involved in the maintenance of endothelium permeability and in the control of the traffic of leukocytes from blood toward inflamed tissues.

Cadherins generally possess a modular structure consisting of five or so Ig-like extracellular cadherin domains, a single-pass transmembrane domain and a conserved cytoplasmic tail. They may be divided into subfamilies according to their sequence identity. Two important subfamilies are the classical type I cadherins such as N-, E-, and C-cadherin, and type II cadherins such as VE- MN- and OB-cadherin. Both these types have a conserved tryptophan (W2) at the $\mathrm{N}$-terminus while the type II cadherins have an additional conserved tryptophan (W4).

The molecular basis of classical type I cadherin homophilic association has been extensively studied. On the basis of considerable biochemical ${ }^{2}$ and structural evidence ${ }^{3},{ }^{4}, 5$, ${ }^{6}$, it is now generally believed that the first extracellular domain (EC1) determines the binding specificity of cadherins ${ }^{7}$. Central to this specificity is the formation of a strand dimer which involves the insertion of tryptophan 2 from EC1 of one molecule into a hydrophobic pocket on $\mathrm{EC} 1$ of the adjacent molecule ${ }^{3}$. Boggon et al. determined the structure of the entire ectodomain of $\mathrm{C}$-cadherin ${ }^{6}$. In this structure, the $\mathrm{ECl}$ trytophan 2-mediated interface is involved in the maintenance of two adjoining cadherin molecules arranged in an almost- 
parallel fashion. The EC1-5 C-cadherin dimer seen in the crystal, is slightly curved with a length of $385 \AA$, and so the dimer cannot be oriented perpendicular to the membrane. It has been proposed that this $\mathrm{C}$-cadherin dimer is oriented at an angle to the membrane to account for the width of the junction and a model based on tomagraphic reconstruction of freeze substituted desmosomes of mouse epidermis has been published ${ }^{8}$. There is no consensus model for the adherens junction. Based on structural evidence, several different models for adherens junctions have been proposed ${ }^{3},{ }^{4}, 5,{ }^{6},{ }^{9},{ }^{8}$. They mostly involve cis-dimerisation of molecules from the same cell and trans-dimerisation of ECl from opposing cells ${ }^{3}$. The cadherin molecules dimerize with a very low binding affinity and it has been suggested that the binding specificity occurs by amplification of small differences of this low affinity binding by multiple cadherin interactions at the adherens junctions ${ }^{\mathbf{1 0}}$.

The structure and mechanism of type II cadherin-mediated adhesion is less well characterised, however, it is thought that the overall 3-dimensional structures and the homophilic interactions of the type I and type II cadherins are similar, despite the low ( $25 \%)$ sequence homology. Electron microscopy data on the extracellular fragment of murine VEcadherin linked to a trimeric base of cartilage matrix protein revealed interactions of the EC1 and EC2 domains similar to type I cadherins ${ }^{11}$. While type I cadherins generally form homotypic interactions, they can interact to some extent with other type I cadherins, but not with type II cadherins ${ }^{\mathbf{1 2}}$. The same holds true for type II cadherins ${ }^{13}$. Also, both the conserved tryptophane (W2 and W4) in the type II cadherins are necessary for junction formation ${ }^{14}$. Thus it is probable that the type II cadherins form a strand dimer which nevertheless differs from that of the type I cadherins.

In contrast to the above structural data, surface force measurements between two monolayers of the complete extracellular domain of C-cadherin, and bead aggregation assays on C-cadherin constructs, suggest that multiple cadherin extracellular repeats mediate 
homophilic self-association of cadherin ${ }^{15},{ }^{16}$. The surface force measurements show three force maxima which have been interpreted to show that three different interdigitating interactions exist. Using several biophysical techniques, we demonstrated that the extracellular part of VE-cadherin forms hexamers in vitro. Cryo-EM of these hexamers revealed a hollow cigar, $233 \AA$ long and $77 \AA$ wide ${ }^{17}$. No dimeric interaction between the VE EC1-2 or EC1-3 fragments in solution was observed while a strong interaction between the EC3-4 fragments was detected ${ }^{18}$.

Here we present a homology model of the VE-cadherin hexamer based on a $3 \mathrm{D}$ reconstruction from negatively stained EM images and the X-ray structure of C-cadherin ${ }^{6}$. This model, consists of a trimer of dimers with each N-terminal EC1 module making an antiparallel dimeric contact and the EC4 modules forming extensive trimeric interactions. It is in accord with the generally held belief that an EC1 dimeric interaction is central to homotypic cadherin interaction. Evidence in favour of the existence of this hexameric architecture in vivo at the adherens junction is examined. This model is used to explain the results of surface force

measurements ${ }^{15},{ }^{16}$ and also to explain the width and striated appearance of recent cryoelectron microscopy of vitreous sections (CEMOVIS) of human skin desmosomes ${ }^{19}$.

\section{Results}

\section{Structure of the VE-EC1-4 he xamer}

Electron micrographs of negatively stained VE-EC1-4 reveal an oligomer in the form of a hollow cigar $233 \AA$ long and $77 \AA$ wide (Figure1(a)) ${ }^{17}$. End-on views in negative stain are occasionally seen in the form of an annulus of 70-80 A diameter (Figure 1(a)). All the single particle image analysis was performed using SPIDER ${ }^{20}$ (Figure 1(b), (c)). The VE-EC1-4 is adsorbed to the carbon with a preferred orientation as seen in Figure 1(e). This is an orientation midway between the 2 -fold axes. There is a ratio of 10 to 1 for images in this 
orientation and images down the 2-fold axis (Figure 1(d)). We note the similarity of this 2fold projection and the averaged image of the hexamer obtained from cryo-electron micrographs of small 2D arrays ${ }^{21}$.

Four distinct regions of density are apparent per asymmetric unit in the reconstruction of the VE-EC1-4 oligomer (Figure 2(a), (b) and (c)). Each domain can be interpreted as an Iglike cadherin module $45 \AA$ long and $25 \AA$ s wide. This produces a hexameric model in accord with our previous biochemical and biophysical studies ${ }^{18}$ which showed that VE-EC1-4 forms a hexamer in solution. The attribution of modules to the four regions of density is unambiguous since the histag on EC4 of the VE-EC1-4-his hexamer binds to a functionalized membrane ${ }^{22},{ }^{21}$ and the modules must be linked to each other in succession.

\section{Fit of a homology model of VE cadherin}

Although little is known about the atomic structure of type II cadherins, they are generally supposed to have a similar structure to the type I cadherins ${ }^{23}$. In particular, the first 4 ectodomains of C-cadherin and VE-cadherin have a homology of only $26 \%$. Nevertheless, a homology model of VE-EC1-4 was predicted based on the crystal structure of C-cadherin ${ }^{6}$. We note that May et al., also used a homology model based on the C-cadherin structure to interpret their data ${ }^{14}$.

The reconstructed map of VE-EC1-4, at 24-Å resolution, allows the homology model of each VE-cadherin module to be positioned visually (Figure 2(d)-(1)). But, there is not sufficient detail to determine the rotational orientation of each module about its long axis (Figure 2(d)-(1)). However, if we suppose that the EC1 dimer, seen in the hexamer, is formed by the exchange of N-terminal Trytophans (W2 and/or W4), as for type I cadherins, then each EC1 must be oriented with its N-terminal strand facing its neighbour (Figure 2(1)).

The hand of the reconstruction has not been determined, but, can be deduced when information concerning the transiently exposed VE-cadherin epitopes on EC1, as described by 
May and colleagues ${ }^{14}$, are also incorporated into the model. Assuming that the hexamer is indeed the oligomer found in native junctions, then the $\mathrm{N}$-terminus of $\mathrm{ECl}$, which contains the epitope of a mAb which is hidden in the intact junction, must be hidden in the hexamer. This condition is already satisfied since we have positioned the N-termini at the EC1-EC1 interface (purple, Figure 2(k)). In addition, the epitope on EC1, which lies within amino acids 45 to $56{ }^{14}$, must be accessible on the hexamer (yellow, Figure 2(k)). This is only the case for the reconstruction with the hand as shown. For the opposite hand, the amino acids 45 to 56 face towards the centre of the hexamer and so would be inaccessible to antibodies.

The density of EC2 is lower than for the other modules. A partial explanation for this may be that the molecule has a torsional flexibility and can unwind to some degree. EC2 may move more than the other modules, at least in rotation. Also, EC2 may be affected by interactions with the carbon film. EC2 lies close to EC1 of the neighbouring three-fold related VE-EC1-4 molecule (cis conformation) and so may interact with EC1 (Figure 2(1)). This is reminiscent of to the EC1-2 cis-dimer interaction seen in the crystal structure of C-cadherin ${ }^{6}$. However, it is clear that the model we have produced cannot give precise interactions between amino acids.

The EC4 modules have extensive trimeric interactions and indeed are the only modules to have such an interaction. EC4 apparently interacts with EC3 on the neighbouring VE-EC1-4. EC1 interacts to form anti-parallel dimers and is the only module to display a trans-dimeric interaction. One full length VE-EC1-4 molecule is estimated to be about $180 \AA$ long, but, since each VE-EC1-4 molecule curves around in a spiral the total length of the hexamer is $230 \AA$.

\section{Discussion}

\section{The EC1 dimerinteraction}


The model we present here for the VE-EC1-4 hexamer consists of a trimer of dimers with each $\mathrm{N}$-terminal EC1 module making a dimeric contact and the EC4 modules forming extensive trimeric interactions. This model is in accord with the generally held belief that an EC1 dimeric interaction is central to homotypic cadherin interaction. The EC1 modules form an anti-parallel dimer. This is a conformation not seen in the crystal structures of type I cadherins. However, this does not preclude the possibility that the dimer is formed by the exchange of the tryptophan(s) of N-terminal strands as for the type I cadherins. Given that the type II cadherins have two conserved tryptophan (W2 and W4), both of which are required for homotypic interaction of VE-cadherin ${ }^{\mathbf{1 4}}$, and type I cadherins have only one conserved tryptophan, it is highly probable that there are differences in the orientation of the EC1 interaction. Also type I cadherins can interact to a certain extent with other type I cadherins but not with type II cadherins ${ }^{24}$. This argues in favour of differences in the cadherin type I and type II interactions. There are differences in the dimeric interactions of EC1 of type I cadherins (C- N- and E-cadherin) seen by X-ray crystallography $\stackrel{6,3,4}{4}$ and it is evident that the N-terminal strand of EC1 can adopt different conformations. The cis-dimer of VE EC1-4 seen in $2 \mathrm{D}$ arrays attached to an isolated monolayer ${ }^{22}$ appears to be a parallel EC1 dimer and thus differs from the dimer seen here. It is possible that VE-cadherin adopts the compact dimer conformation at the surface of an isolated cell for protection from degradation and only forms the hexamer at the cell-cell junction.

In EM studies of VE-cadherin Engel and colleagues ${ }^{11}$ demonstrated the dimerisation of the ectodomains VE-EC1-5 via EC1 and/or EC2. VE-EC1-5 was connected by linker sequences to the $\mathrm{N}$ terminus of the trimeric cartilage matrix protein (CMP). This means that the "local" concentration of VE-EC1-5 at each CMP is very high. Dimers of VE-EC1-5 were seen as rings on the same CMP (cis-dimer), and at higher $\mathrm{Ca}^{++}$concentration double rings, or trans-dimers, were visible, but it is of note that no dimers were seen to form directly between 
VE-EC1-5 from different CMP. These results indicate that the dimeric interaction only occurs at high concentration, or when more than one dimeric interaction can occur (cooperativity). These results help to explain the apparent lack of dimer formation of our isolated VE-EC1-4 fragment in solution ${ }^{\mathbf{1 8}}$, but the formation of dimeric interactions in the hexamer where the cooperative effect of multiple dimeric interactions is present. Only at high VE-EC1-4-His concentration at the lipid monolayer did we see dimer formation ${ }^{22}$. Engel and colleagues did not see any hexamers. It is quite possible that the VE-EC1-5 grafted to CMP is not rotationally free to form trimers via the EC4 module and so cannot form hexamers.

\section{Alternative explanation of force mea surement between cadherin extracellular domains}

In contrast to most data on cadherin interactions, surface force experiments have been interpreted as showing that several EC domains are involved in the homotypic interaction ${ }^{\mathbf{9}},{ }^{\mathbf{2 5}}$.

In these experiments the extra-cellular domains of $\mathrm{C}$-cadherin are bound to opposing surfaces via a C-terminal 6-His tag. The force between these cadherin-covered surfaces is measured as they are brought together and then separated. At three discreet distances force maxima are observed and have been interpreted to indicate that different cadherin modules contribute to adhesion with the formation of inter-digitating structures in three different overlapping conformations at inter membrane separations of 250,320 and $400 \AA$. The hexameric model we present here provides an alternative explanation for these results, supposing that the Ccadherin also forms hexamers. As the surfaces are pulled apart we suppose that first one dimeric bond per hexamer is broken and the molecule unwinds slightly, similarly for the second and finally rupture of the interface occurs when the third dimer is broken. In a previous study designed to explore the role of each VE-EC domain in the formation of the VE-cadherin interaction we found a strong interaction between the EC3-4 fragments but found no interaction between the EC1-2 and the EC1-3 fragments. As described above we suppose that the dimer interaction is very weak and only forms in the context of the hexamer 
where the cooperative effect of multiple dimer interactions is present. It is of note that the model proposed here with a cis-trimer of trans-dimers gives a stronger bond due to cooperativity. In this model the EC4-EC4 interaction plays an important role in strengthening the cell-cell bond. This is in accord with previous work which demonstrated the importance of modules, other than EC1, in the formation of the cadherin bond ${ }^{\mathbf{2 6}},{ }^{\mathbf{1 2}}$.

\section{Does the hexamer exist in vivo?}

Cryo-electron microscopy of vitreous sections (CEMOVIS) is currently the best method of observing sections of tissue in their fully hydrated native state ${ }^{27}$. The images display more detail and a better resolution than electron micrographs of conventionally prepared sections where dehydration can cause aggregation and distortion of the specimen. The micrographs of CEMOVIS of human skin desmosomes ${ }^{19}$ reveal the cadherin junction to be $290 \AA$ wide and show strands roughly perpendicular to the membrane with a repeat distance of the order of $50 \AA$ with a darker band at the mid line. This shows remarkable resemblance to the artificial adherens junctions formed by the VE-EC1-4-His hexamer at the surfaces of liposomes and observed by cryo-EM ${ }^{21}$. These artificial junctions are $230 \AA$ wide (the length of the hexamer) and display $40 \AA$ striations roughly perpendicular to the membrane. We suppose that the EC5 module continues the spiral in the native junction. Five cadherin modules laid out straight are roughly $225 \AA$ long, so two cadherin ectodomains end to end measure $450 \AA$, which is much greater than the width of an adherens junction. The hexameric model composed of highly curved cadherin molecules, which nevertheless appears to have parallel strands when viewed side-on, provides an explanation for both the width and the striations of the junction seen by CEMOVIS.

The different cadherins may or may not form junctions with the same architecture. The hexamer model described here can explain much of the existing data on cadherin interactions in general and so may be a common motive. However, direct evidence of the 
existence of the hexamer at the adherens junction is lacking. A tomographic reconstruction of an adherens junction seen by CEMOVIS would help to resolve this question.

\section{Materials and mothods}

\section{Expression and purification of the recombinant fragment VE-EC1-4}

Expression and purification of the recombinant fragment VE-EC1-4 was carried out as described $^{\mathbf{1 8}}$.

\section{Electron microscopy and image analysis}

Samples of VE-EC1 -4 negatively stained with $2 \%(\mathrm{w} / \mathrm{v})$ uranyl acetate were examined using a Philips CM12 electron microscope equipped with a $\mathrm{LaB}_{6}$ filament operating at $120 \mathrm{kV}$. The micrographs were recorded under low dose conditions $\left(<20\right.$ electrons $\left./ \AA^{2}\right)$ at a nominal magnification of 45,000X. Micrographs were selected for image quality and then digitized using a Zeiss SCAI scanner with a $14 \mu \mathrm{m}$ step size which corresponds to a nominal pixel size of $3.21 \AA$. Six micrographs with a defocus such that the first zero of the CTF was beyond 22 $\AA$ were analyzed. Images of individual particles were selected with $\mathrm{x} 3 \mathrm{~d} 2$ and the subsequent image analysis was made with the program suite SPIDER ${ }^{20}$. A total of 1350 particles were selected, and were normalized to the same mean and standard deviation, without CTF correction, with SPIDER. Ten different starting models were generated from single images or from a reference free class average, with a symmetry axis imposed along the long axis. Twothree- and four-fold symmetry axes were tested but only the 3-fold axis produced an interpretable reconstruction. Additional 2-fold axes were apparent on the equatorial plane and were subsequently introduced to give D3 symmetry. The resolution was determined by Fourier shell correlation (50\% value) to be $24 \AA$.

\section{Homology modelling of VE EC1-4}


A homology model for the first 4 modules of VE-cadherin, based on the structure of the Ccadherin EC1 $-5{ }^{6}$, was generated using the program modeller ${ }^{28}$. The sequence alignment was made using CLUSTAL ${ }^{29}$. The homology model of each VE cadherin module was positioned visually in the reconstructed map of VE-EC1-4 map using "O" ${ }^{30}$.

Figures 1(b)-(f) were made using SPIDER ${ }^{20}$. Figures 2(a)-(c) were produced using Amira (Mercury Computer Systems Courtaboeuf, France), and Figures 2(d)-(1) were made with PyMOL (http://pymol.sourceforge.net/)

\section{Acknowledgements}

We thank Frederic Metoz for assistance in running the computers, Jorges Navazza, Helen Saibil and Guy Schoehn for discussions. This work was supported by grants from CNRS (ACI PCV and DRAB).

\section{Figure Captions}

Figure 1: Electron micrographs of VE-EC1 4 negatively stained with 2\% uranyl acetate.

(a). Side views. INSET End-on views. The scale bar represents $200 \AA$.

(b) shows characteristic averaged images and (c) shows the corresponding projections of the VE-EC1-4 hexamer reconstruction. (d) is an enlarged projection down a 2-fold axis, (e) is a projection mid-way between two 2-fold axes and (f) shows an end-on projection.

Figure 2: Surface representations of the VE-EC1-4 hexamer.

(a) is a view down a 2-fold axis, (b) is in an arbitrary orientation and (c) is a top view.

(d) Stereo view down a 2-fold axis of the homology model with the VE-EC1-4 dimers represented in violet, light-green and light-blue.

(e) to (l) represent the fit of the VE-EC1-4 homology model in the reconstructed hexamer. EC1 (red), EC2 (green), EC3 (cyan) and EC4 (blue) are coloured as indicated. (e) is a stereo 
view midway between two 2-fold axes showing the fit of one dimer of VE-EC1-4. In (f) all six VE EC1-4 molecules are present. (g), (h), (i), and (j) are views down the long axis of thick sections each chosen to include only one EC module. (k) is a closer view of the EC1-EC1 dimer showing the N-terminal epitope (residues 4-10, purple) and the epitope (residues 45-56, yellow $)^{\mathbf{1 4}}$. In (I) a cis-dimer interaction between EC1-EC2 on neighbouring VE-EC1-4 molecules is indicated by an asterisk. Note the similarity with the same EC1-EC2 dimer interaction seen in the C-cadherin crystal structure ${ }^{6}$.

\section{References}

1. Kemler, R. (1992). Classical cadherins. Semin Cell Biol 3, 149-55.

2. Koch, A. W., Pokutta, S., Lustig, A. \& Engel, J. (1997). Calcium binding and homoassociation of E-cadherin domains. Biochemistry 36, 7697-705.

3. Shapiro, L., Fannon, A. M., Kwong, P. D., Thompson, A., Lehmann, M. S., Grubel, G., Legrand, J. F., Als-Nielsen, J., Colman, D. R. \& Hendrickson, W. A. (1995). Structural basis of cell-cell adhesion by cadherins [see comments]. Nature 374, 32737.

4. Nagar, B., Overduin, M., Ikura, M. \& Rini, J. M. (1996). Structural basis of calciuminduced E-cadherin rigidification and dimerization. Nature 380, 360-4.

5. Pertz, O., Bozic, D., Koch, A. W., Fauser, C., Brancaccio, A. \& Engel, J. (1999). A new crystal structure, $\mathrm{Ca} 2+$ dependence and mutational analysis reveal molecular details of E-cadherin homoassociation [In Process Citation]. EMBO J 18, 1738-47.

6. Boggon, T. J., Murray, J., Chappuis-Flament, S., Wong, E., Gumbiner, B. M. \& Shapiro, L. (2002). C-cadherin ectodomain structure and implications for cell adhesion mechanisms. Science 296, 1308-13.

7. Nose, A., Tsuji, K. \& Takeichi, M. (1990). Localization of specificity determining sites in cadherin cell adhesion molecules. Cell 61, 147-55.

8. He, W., Cowin, P. \& Stokes, D. L. (2003). Untangling desmo somal knots with electron tomography. Science 302, 109-13.

9. Leckband, D. \& Sivasankar, S. (2000). Mechanism of homophilic cadherin adhesion [In Process Citation]. Curr Opin Cell Biol 12, 587-92.

10. Chen, C. P., Posy, S., Ben-Shaul, A., Shapiro, L. \& Honig, B. H. (2005). Specificity of cell-cell adhesion by classical cadherins: Critical role for low-affinity dimerization through beta-strand swapping. Proc Natl Acad Sci US A 102, 8531-6.

11. Ahrens, T., Lambert, M., Pertz, O., Sasaki, T., Schulthess, T., Mege, R. M., Timpl, R. \& Engel, J. (2003). Homoassociation of VE-cadherin follows a mechanism common to "classical" cadherins. J Mol Biol 325, 733-42.

12. Shan, W., Yagita, Y., Wang, Z., Koch, A., Svenningsen, A. F., Gruzglin, E., Pedraza, L. \& Colman, D. R. (2004). The minimal essential unit for cadherin-mediated intercellular adhesion comprises extracellular domains 1 and 2. J Biol Chem 279, 55914-23. 
13. Shimoyama, Y., Tsujimoto, G., Kitajima, M. \& Natori, M. (2000). Identification of three human type-II classic cadherins and frequent heterophilic interactions between different subclasses of type-II classic cadherins. Biochem J349, 159-67.

14. May, C., Doody, J. F., Abdullah, R., Balderes, P., Xu, X., Chen, C. P., Zhu, Z., Shapiro, L., Kussie, P., Hicklin, D. J., Liao, F. \& Bohlen, P. (2005). Identification of a transiently exposed VE-cadherin epitope that allows for specific targeting of an antibody to the tumor neo vasculature. Blood 105, 4337-44.

15. Sivasankar, S., Brieher, W., Lavrik, N., Gumbiner, B. \& Leckband, D. (1999). Direct molecular force measurements of multiple adhesive interactions between cadherin ectodomains. Proc Natl Acad Sci US A 96, 11820-4.

16. Sivasankar, S., Gumbiner, B. \& Leckband, D. (2001). Direct measurements of multiple adhesive alignments and unbinding trajectories between cadherin extracellular domains. Biophys $J 80,1758-68$.

17. Legrand, P., Bibert, S., Jaquinod, M., Ebel, C., Hewat, E., Vincent, F., Vanbelle, C., Concord, E., Vernet, T. \& Gulino, D. (2001). Self-assembly of the vascular endothelial cadherin ectodomain in a $\mathrm{Ca} 2+$-dependent hexameric structure. $J$ Biol Chem 276, 3581-8.

18. Bibert, S., Jaquinod, M., Concord, E., Ebel, C., Hewat, E., Vanbelle, C., Legrand, P., Weidenhaupt, M., Vernet, T. \& Gulino-Debrac, D. (2002). Synergy between extracellular modules of vascular endothelial cadherin promotes homotypic hexameric interactions. J Biol Chem 277, 12790-801.

19. Al-Amoudi, A., Dubochet, J. \& Norlen, L. (2005). Nanostructure of the epidermal extracellular space as observed by cryo-electron microscopy of vitreous sections of human skin. J Invest Dermatol 124, 764-77.

20. Frank, J., Radermacher, M., Penczek, P., Zhu, J., Li, Y., Ladjadj, M. \& Leith, A. (1996). SPIDER and WEB: processing and visualization of images in 3D electron microscopy and related fields. J Struct Biol 116, 190-9.

21. Lambert, O., Taveau, J. C., Him, J. L., Al Kurdi, R., Gulino-Debrac, D. \& Brisson, A. (2005). The basic framework of VE-cadherin junctions revealed by cryo-EM. $J$ Mol Biol 346, 1193-6.

22. Al-Kurdi, R., Gulino-Debrac, D., Martel, L., Legrand, J. F., Renault, A., Hewat, E. \& Venien-Bryan, C. (2004). A soluble VE-cadherin fragment forms 2D arrays of dimers upon binding to a lipid monolayer. J Mol Biol 337, 881-92.

23. Nollet, F., Kools, P. \& van Roy, F. (2000). Phylogenetic analysis of the cadherin superfamily allows identification of six major subfamilies besides several solitary members. J Mol Biol 299, 551-72.

24. Shan, W. S., Tanaka, H., Phillips, G. R., Arndt, K., Yoshida, M., Colman, D. R. \& Shapiro, L. (2000). Functional cis-heterodimers of N- and R-cadherins. J Cell Biol 148, 579-90.

25. Leckband, D. (2001). Force as a probe of membrane protein structure and function. Curr Opin Struct Biol 11, 433-9.

26. Chappuis-Flament, S., Wong, E., Hicks, L. D., Kay, C. M. \& Gumbiner, B. M. (2001). Multiple cadherin extracellular repeats mediate homophilic binding and adhesion. $J$ Cell Biol 154, 231-43.

27. Al-Amoudi, A., Norlen, L. P. \& Dubochet, J. (2004). Cryo-electron microscopy of vitreous sections of native biological cells and tissues. J Struct Biol 148, 131-5.

28. Fiser, A. \& Sali, A. (2003). Modeller: generation and refinement of homology-based protein structure models. Methods Enzymol 374, 461-91.

29. Higgins, D. G. \& Sharp, P. M. (1988). CLUSTAL: a package for performing multiple sequence alignment on a microcomputer. Gene 73, 237-44. 
30. Jones, T. A., Zou, J. Y., Cowan, S. W. \& Kjeldgaard. (1991). Improved methods for building protein models in electron density maps and the location of errors in these models. Acta Crystallogr A 47 ( Pt 2), 110-9. 
Dear Sir,

We submit here our manuscript entitled "Architecture of the VE-cadherin hexamer" by Elizabeth A Hewat, Claire Durmort, Lilian Jacquamet, Evelyne Concord and Danielle Gulino-Debrac, for publication in Journal of Molecular Biology.

The model we present here for the vascular endothelial-cadherin (VE-cadherin) hexamer throws a new light on cadherin interactions. It is in accord with the generally held belief that a dimeric interaction of the $\mathrm{N}$-terminal cadherin module is central to homotypic cadherin interactions, but involves an additional trimeric interaction of the 4th extracellular module. It provides a novel explanation for force measurements of the interaction between opposing cadherin layers, and for cryo-EM observations of native junctions. In the context of the cell-cell junction this hexamer with cis-trimers close to the membrane and trans-dimers from opposing membranes, increases the avidity of the bond. The fact that this hexameric model of VE-cadherin can be used to explain more of the existing data on adherens junctions than any other model alone argues in favour of the existance of the hexamer at the adherens junction.

Yours sincerely, Elizabeth Hewat

\section{Suggested reviewers are:-}

Dr Phoebe L. Stewart

Molecular \& Medical Pharmacology

A-324 Crump Inst.

Box 951770

Los Angeles, CA 90095-1770

USA

Email: pstewart@mednet.ucla.edu

Office: A-324C Crump Inst.

Phone: 310-206-7055

Lab: A-324 Crump Inst.

Phone: (310) 206-7054

Dr Bettina Bottcher

Structural and Computational Biology Programme, European Molecular Biology Laboratory, Meyerhofstrasse 1, 69117 Heidelberg, Germany. Tel.: 49-6221-387304; 
Fax: 49-6221-387265;

E-mail: boettcher@embl.de.

Dr. Alasdair C. Steven

Laboratory of Structural Biology Research

NIH, Building 6, Room 425

6 Center Drive MSC 2755

Bethseda, Maryland 20892-2755

USA

Tel: (301) 4043418

Fax: (301) 4043417

E-mail: steven@calvin.niams.gov

Dr. Laurence Shapiro

Department of Biochemistry and Molecular Biophysics,

Columbia University,

630 West 168th Street

New York, NY 10032,

USA

Tel: +1 (212) 342-6029

Fax: +1 (212) 342-6026

Email: shapiro@convex.hhmi.columbia.edu

Dr. Helen Saibil

Department of Crystallography

Birkbeck College

Malet Street

London WC1E 7HX

UK

Tel: (44) 01716316820

Fax: (44) 01714368918

E-mail: h.saibil@mail.cryst.bbk.ac.uk

Dr. Deborah Leckband

Center for Biophysics and Computational Biology,

University of Illinois

Urbana-Champaign,

Urbana, Illinois,

USA

TEL: 0001 217-244-0793

FAX: 0001 217-333-5052

E-mail: leckband@uiuc.edu

Please note we would rather not have

Dr. Elisabetta Dejana

Milan, Italy

as a referee. Thank you. 
Figure 1

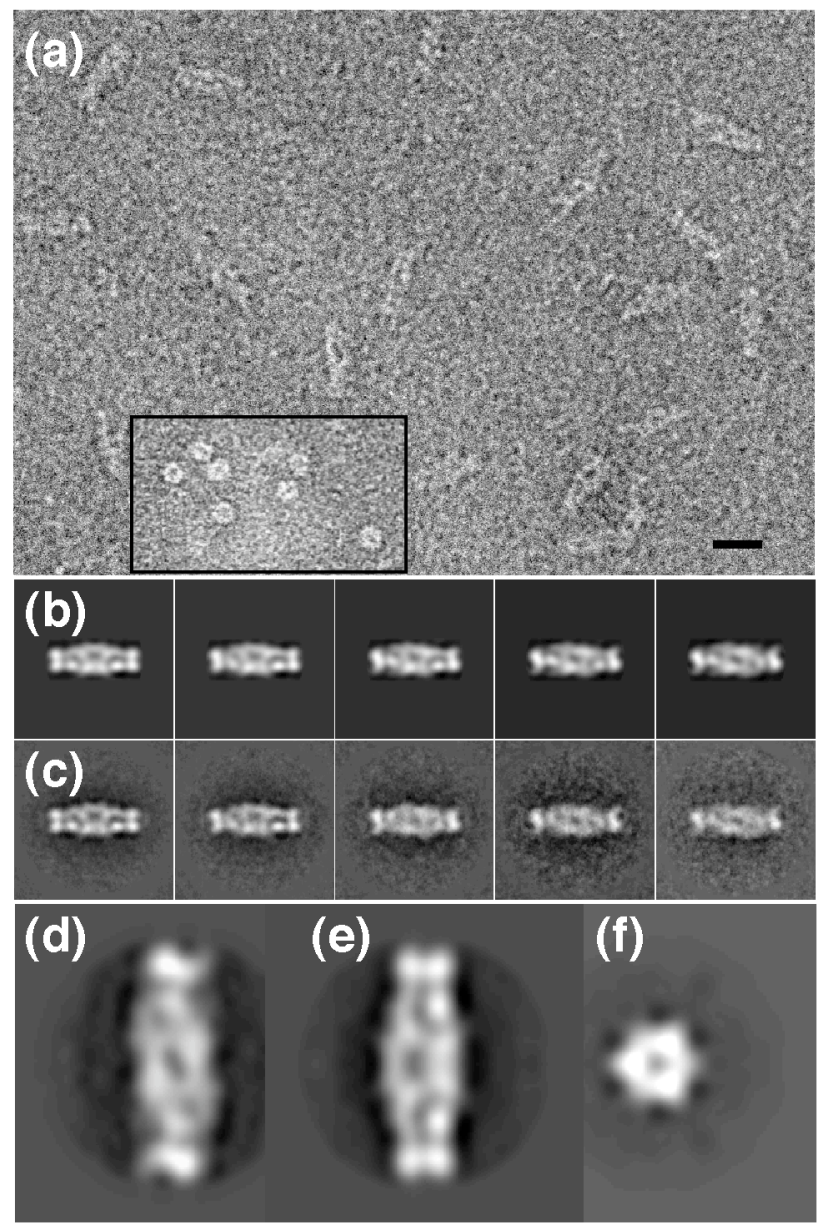

Hewat et al. Figure 1 
(a)

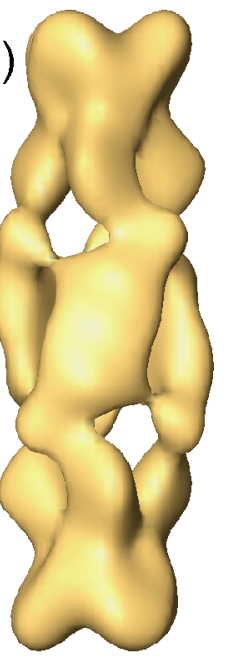

(e)

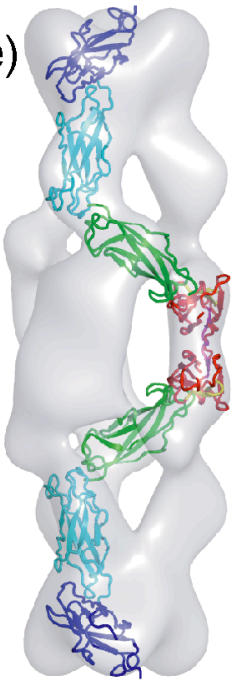

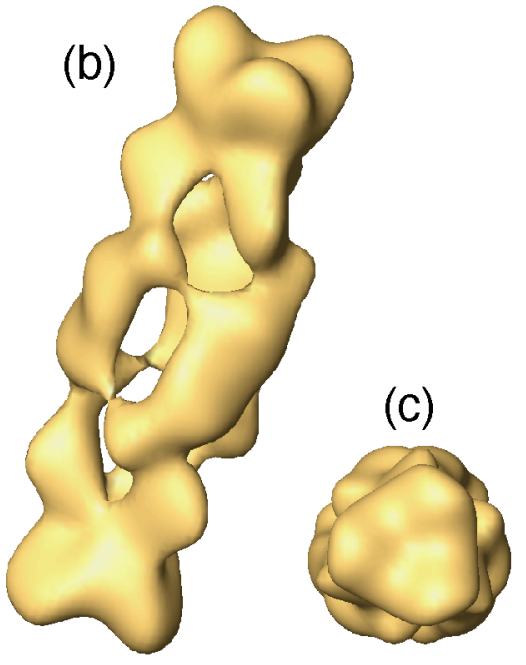

(d)

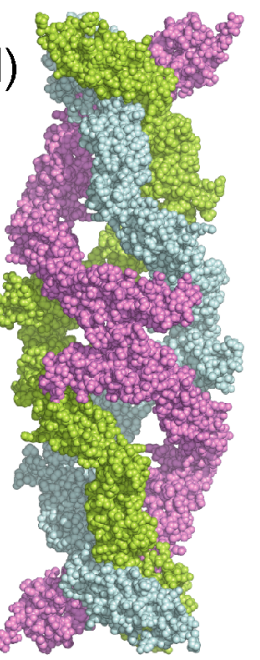

(f)
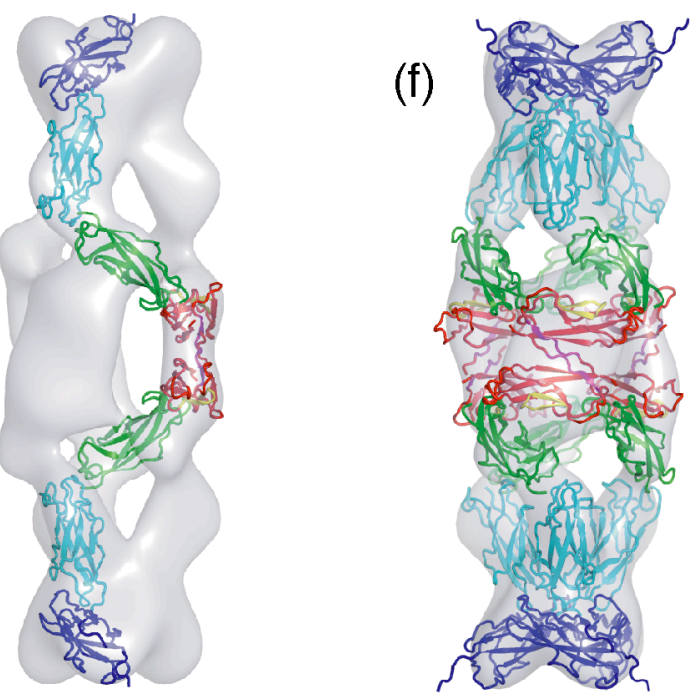

(k)

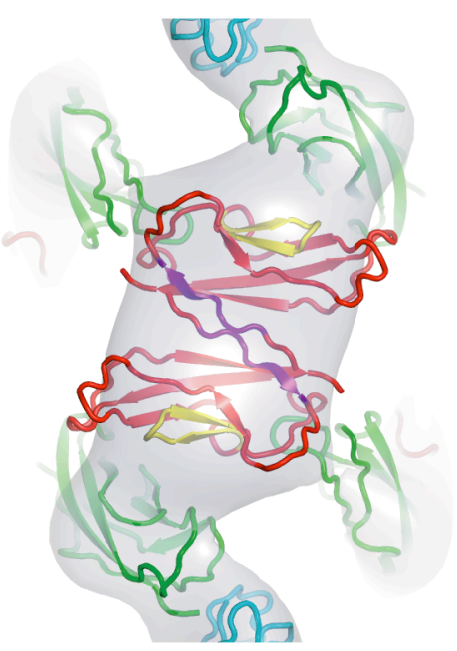

(I)

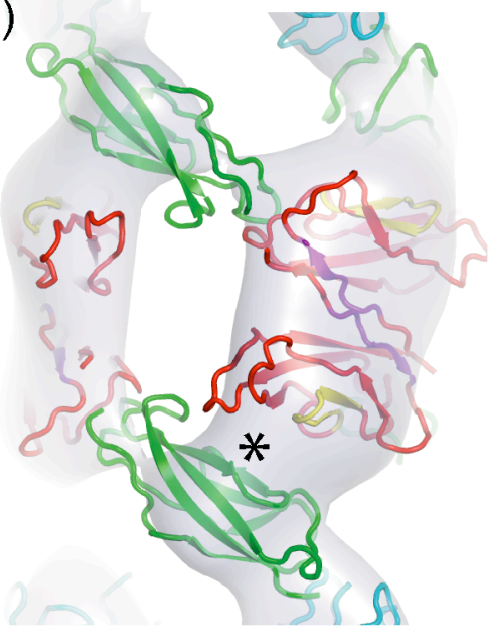

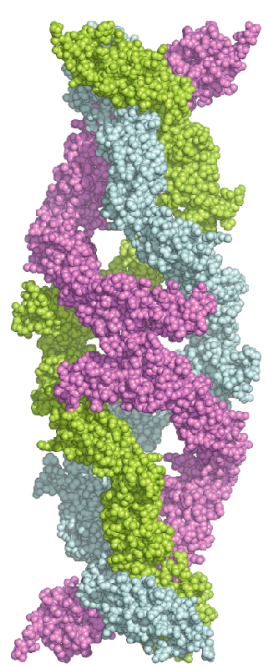

(g)

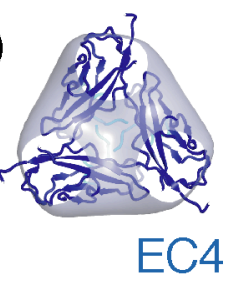

(h)

(t)

A. 15

EC3

(i)

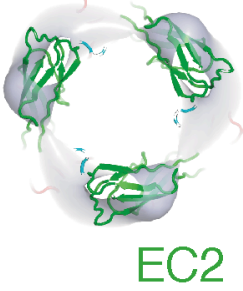

(j)

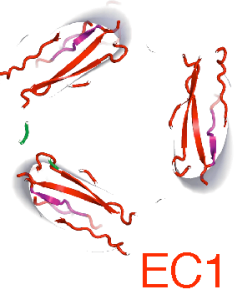

